\title{
Reflexones marxinales sobro sociollingüística asturiana ${ }^{\mathrm{I}}$
}

\author{
por Xulio Viejo Fernández \\ Universidá d'Uviéu
}

\begin{abstract}
T a investigación sociollingüística n’Asturies vieno a desenvolvese Lal par del movimientu de reivindicación llingüística de les últimes décades. Nesti contestu, usada como ferramienta d'análisis pero tamién como provisora d'espectatives y compromisu pa cola realidá descrita, la sociollingüística asturiana quiciabes tien acusao esa urxencia d'apurrir argumentos pal debate sobro polítiques llingüístiques ensin ser quien a afitar un marcu de referencia coherente pa encetar les cuestiones llatentes sobro la esistencia social del asturianu.

De primeres, una sociollingüística asturiana había empezar por definir el marcu variacional de los usos llingüísticos del país, en considerando tanto les claves de l'articulación interna de les variedaes y rexistros como'l contactu y amiestu col castellanu. Neses coordinaes, afayaríen el

${ }^{1}$ Esti artículu foi publicáu orixinariamente n’euskera col títulu «Bazterreko gogoetak Asturiaseko soziolinguistikaren inguruan", Bat. Soziolinguistika Aldizkaria, I0I/4 (2016), páxs. I5I-I68. Esta versión reproduz lliteralmente'l testu orixinal, col amiestu de delles precisiones menores pero pertinentes pal públicu asturianu, non presentes nel orixinal por razones editoriales. Quiero agradece-yos a los responsables de la revista Bat la so autorización pa la reproducción d'esti artículu, mesmo que’l so interés pola realidá social de la llingua asturiana.
\end{abstract}


so sentíu últimu otres considerances relatives a les actitúes reconocibles nesi ecosistema llingüísticu, incluyida ende la percepción social de los modelos normativos o testuales que quieren volvese referenciales nel procesu de normalización y que (d'acordies col so nivel d'aceptación pela parte del falante) condicionen abondo la empatía hacia la reivindicación llingüística.

Delles d'estes cuestiones foron trataes dende bien primero de la formulación de la teoría sociollingüística, lo que da idea de la so fondura histórica y de la so trescendencia na concencia llingüística asturiana. Dende'l xvi, autores como Juan de Valdés o Luis Alfonso de Carvallo yá dexen ver el surdimientu del castellanu como llingua de prestíu y el retrocesu o corrupción del asturianu a partir d'una imaxe más o menos idealizada de la so supuesta pureza orixinal. A otru sen, una obra teatral d'Antón de Marirreguera en Xvir fai rescamplar una asociación clara ente llingua ya identidá social, al confrontar l'asturianu de los personaxes populares col castellanu redichu y risible de quien pon a asoleyar col so emplegu un falsu estatus (Viejo, 2002).

Un sieglu depués, Xovellanos fai una descripción más esplícita de l'amestadura col castellanu nes ciudaes del so tiempu (Andrés Díaz, 2002: 32) y acredita una percepción unitaria del asturianu percima de les sos variedaes locales (Xovellanos, I98r: 68). En I839 Xosé Caveda volvía aportunar nel so retrocesu n'ámbitos urbanos y ente la mocedá aguiyáu por cuestiones como la educación, el serviciu militar o la emigración. Caveda asume que l'asturianu llegó hasta'l so tiempu «sin alteraciones sensibles que desfiguren su antigua índole»; sicasí (y esti ye’l filtru ideolóxicu de les élites del XIX), "no a la sociedad de nuestros días corresponde, sino a otra muy apartada de ellos, y menesterosa en extremo de cultura y buen gusto». Por eso, "aquellas voces y frases antes comunes a toda la provincia, actualmente se usan solo en los lugares y caseríos distantes de los pueblos agregados», anque "todavía en las aldeas se conserva» (Cave- 
da, I989: I-2, 59-60), lo que, otra manera, nun yera poco nun momentu de mayoría descomanada de población rural.

Los primeros estudios dialectales van poner énfasis na variación xeográfica del asturianu, dacuando con formulaciones de llargu recorríu nel debate posterior, al cuestionar dacuando la so integridá en cuenta d'una fragmentación babélica malapenes superable por mor del advenimientu del castellanu. El primer plantegamientu d'esti tipu débese-y al suecu Åke Wilhelmson Munthe, qu'en I887 publica un estudiu sobro la variedá occidental nel que pon de relieve la espansión del castellanu inclusive nes villes pequeñes cabeceres de conceyu, l'atomización del asturianu y distintos niveles nel usu local al son de la distinta receptividá pa coles traces espansives de la llingua oficial (Munthe, I987: I5-I6)².

Anque de manera más o menos sesgada, estos autores tán dando cuenta de procesos sociales que, de xuru, empezaben dase daquella. Otro ye la fuerza real d'esta castellanización, el so ritmu, les sos formes de penetración y el caláu de los discursos y actitúes sociales al respectu en distintos entornos.

Ello ye que les descripciones dialectales d'autores locales van apurrir una visión enforma más matizada. Menéndez Pidal, en 1906, describe la situación n’Asturies (n’oposición a la esistencia dialectal de les variedaes meridionales, percibíes ensin más como castellano mal hablado) como un billingüismu diglósicu asemeyáu al del gallegu (García, 2008). Pidal nun cuestiona la unidá del asturianu y, entá más, xunto a una descripción refecha de la so variación, apunta (nuna encuesta inédita de I9II) la

${ }^{2}$ A esti sen, Munthe refierse a la relativa pureza de la fala de los vaqueiros que "parece estar (...) más libre de mezcla castellana que lo que parece ser general en la lengua de los hablantes fijos, al menos en la cercanía de las ciudades y las carreteras, sin duda una consecuencia del relativo aislamiento en el que parecen vivir estos pastores». Esti testimoniu contrasta vivamente col que malapenes un sieglu primero diera Xovellanos, pal que «la lengua de los vaqueiros es enteramente la misma que la de todo el pueblo de Asturias». 
penetración contra les ales de soluciones asturiano-centrales qu'algamen asina bonalmente un valir referencial y unificador del continuu.

Esta llectura de los datos pidalianos de I9II débese-y propiamente a Diego Catalán nos sos trabayos de los cincuenta sobro l'occidente d'Asturias (I956: 90) que, en xunto colos de Rodríguez-Castellano (I954) y Menéndez García (1963), certifiquen con curtios apuntes sociollingüísticos tanto la vitalidá de la fala local como les influencies uvieínes, a la tema coles del castellanu oficial, que frecuentemente yera vistu como un rexistru afectáu, en clave asemeyada a la que reflexara'l teatru de Marirreguera trés sieglos primero.

L'exerciciu más notable de sociollingüística avant la lettre fírmenlu Catalán y Galmés en 1946 a la d'estudiar l'aspiración de /f/ nel oriente d'Asturies. De la que lo faen, describen la penetración del castellanu y, sobro manera, del asturianu central pelos llugares meyor comunicaos y pente les xeneraciones nueves o con meyor instrucción. Sicasí, esti enfoque innovador malapenes tuvo continuidá, bien que cuestiones como la unidá de la llingua o la so falta de prestíu tienen sío los grandes tópicos del debate social sobro l'asturianu.

Delles claves y términos de la naciente sociollingüística aprucen pela primer vez en 1967 nun trabayu de Josefina Martínez que caracteriza la situación de la capital mientres el Franquismu como de diglosia asturianu-castellanu, analiza l'amiestu d'entrambos códigos y contrapón el modelu más acastellanáu de la ciudá col altu grau de pervivencia del asturianu na so periferia. Sicasí, van pasar más de dos décades hasta qu'una investigación s'afaiga dafechu a los métodos sociollingüísticos: la de Ramón d'Andrés (Andrés, 1993) sobro la rodiada periurbana de Xixón.

De magar, son de considerase otru tipu de trabayos de distintos enfoques y entidá qu'entá nun foron a constituyir un corpus homoxeneu nin a definir un discursu consistente sobro les claves sociollingüístiques 
asturianes. Más que pola sociollingüistica de la llingua, la investigación de los últimos años paez tener apostao polo relativo a la socioloxía del llinguaxe: les actitúes sociales pa cola llingua, cuando con perspectiva histórica (Rodríguez Valdés, 2009) o aplicada (González Riaño, I994). Sía comoquier, les principales referencies son de naturaleza política: encuestes como diagnósticu d'usu y actitúes sociales tocantes a la llingua y les polítiques llingüístiques. Fai'l casu de la publicada por SADEI en I984 y les entamaes por Llera Ramo en I99I, 2002 y 2005.

Ello ye que bona parte del debate públicu sobro la llingua encontóse estos años nos datos ya interpretaciones d'estos trabayos garrando les fríes estimaciones cuantitatives sobre vezos y actitúes como sofitu de la reivindicación, cuando cuestionando la validez de les sos conclusiones o métodos. Por casu, el sofitu mayoritariu a la oficialidá que se deduz de les encuestes invócase como fonte de llexitimidá democrática na reivindicación llingüística y, pela cueta, el desaxuste ente elles y los sucesivos resultaos electorales (que beneficien a partíos contrarios a la oficialidá) val d'argumentu a la de faeles de menos, siendo obvio que la validez d'un estudiu d'esti ser nun ye de dilucidase nestos términos.

Hai entós riesgu de da-y al estudiu sociollingüísticu un sen militante (o, por dicir, compromisivu) que pase del ser del puru suministru de datos útiles pa l'acción social a un tener que ser que de toes toes nun puede faer parte del trabayu científicu, por cuantes que, cuando se pasa d'elaborar un discursu a posteriori sobro unos datos a un prexuiciu que trescala procedimientos y métodos, comprométese seriamente la solvencia de les conclusiones. D'esta miente, en 2006 la Academia de la Llingua Asturiana entamó una encuesta en zones llinderes de Lleón (González Riaño \& García Arias, 2006) en cata d'elementos de continuidá con Asturies no que fai a concencia llingüística. Formulábense dos preguntes: «La manera de hablar de aquí ¿se parece a la de algunas zonas de Asturias?» y «El habla tradicional de la zona ¿es distinta del 
castellano?». La primera suma 176 «muy de acuerdo» y I25 «bastante de acuerdo" sobre 400; la segunda 223 y 8I, respectivamente. Esto empobina a la conclusión d'una identificación mayoritaria ente los lleoneses cola llingua de los asturianos y un alloñamientu respecto al castellanu; pero, cuciamente, bien podía suponese que si les preguntes se fixeren al revés el mesmu falante podía bonamente dar cuenta d'una alta afinidá col castellanu y de que, coles mesmes, la so forma de falar estrémase de la de los asturianos por un determináu númberu de diferencies oxetives.

A última hora, esti tipu de desaxustes son posibles pola ambigua percepción qu'entá se tien del asturianu y el so usu y, polo tanto, poles carencies apuntaes en materia de sociollingüistica de la llingua. Reclamábase enantes un marcu de referencia claru con una estimación razonable del númberu de falantes de la llingua estudiada, que vien ser la cuestión a la que s'aplicaron les encuestes citaes. Sicasí, ello quier esclariar una incógnita previa: ¿qué ye falar asturiano?, y eso a un doble sen: al de la pura caracterización tipolóxica que nos apurre la llingüística internista y el propiamente sociolóxicu que tien consideranza del so usu consciente ya intencional en contestu como pauta de socialización contrastiva con respecto al usu del castellanu. D'estes cuestiones malapenes s'ocuparon con daqué métodu Andrés Díaz (2002) y Fernández Lorences (20II). N'otru tipu d'análisis cuantitativos la noción de lo que ye socialmente falar asturiano malapenes se remana de manera intuitiva y ambigua.

Esto, quiciabes malo d'entender de fora del ámbitu asturianu, esplícase dientro d'él a cuenta de dos variables: una clara concencia histórica de falar daqué propio definío por contraste col castellanu, contrapuntiada por una situación real d'amestadura ente les dos llingües (mayoritaria y espansiva) na que la delimitación d'estes sobro criterios formales da en ser un exerciciu más bien voluntarista pal falante común. Son cuestiones qu'afecten la propia metodoloxía de la encuesta y son quien a esplicar que les dos más solventes (les de I991 y 2002) acaben apurriendo resul- 
taos bien diferentes inclusive pa los mesmos grupos xeneracionales. La primera ofrez datos enforma más optimistes: si ente los nacíos ente la Posguerra y la Transición l'asturianu yera reconocíu en I99i como llingua propia mayoritaria, en 2002 yá da en ser minoritariu nos mesmos grupos etarios. Más qu’un desgaste llingüísticu insólitu ye de pensar que l’inquiz d'esti desvíu tenga que ver tanto cola evolución de la percepción social de la llingua como cola forma de midila.

No que fai a lo último, en I99I, al preguntar pola primer llingua, danse d'opciones «asturiano» (36\%), «castellano» (32\%), «las dos» (6\%), «una mezcla» $(24 \%)$ y «otras»3. Con estes, hasta un $66 \%$ tendría como primer llingua (mutatis mutandis) l'asturianu. Pela cueta, como «lengua de expresión más fácil», el castellanu xube al $47 \%$, l'asturianu queda en $30 \%$, «ambas» en $16 \%$ y «depende» pal $6 \%$. Polo tanto, los de la opción «mezcla» pasen mayoritariamente al castellanu, inchendo tamién una parte pequeña la opción billingüe, ente que l'asturianu pierde porcentaxe. ¿Cómo percibe entós el falante esa «mezcla»? D’Andrés caracteriza’l rexistru mediu de les ciudaes como un Asturianu Minimu Urbanu que, anque llueñe del ideal normativu, entá se percibe inequívocamente como asturianu, frente a variedaes contactuales más arralaes teníes como castellanu asturianizao. Sicasí, d'acordies colos datos de I99I, del casi cuartu de población que diz falar una «mezcla», malapenes la metá paez movida a integralo nun conceptu mínimu d'asturianu.

En 2002, la opción «mezcla» desapaez del cuestionariu y los resultaos pa «primera lengua» (desdoblada en «lengua familiar» $\mathrm{y}$ "lengua en que se empezó a hablar») apurren unes medies que van dende’l $47 \%$ pal castellanu nos nacíos primero de 1942 , con $27 \%$ pal asturianu, y $23 \%$ pa

3 El precedente más inmediatu, la encuesta de SADEI conformábase en 1985 con estremar los que falaben castellano con acentu asturianu (un 48,6 \%) de los que falaben bable o asturiano (un $29, \mathrm{I} 3 \%$ ). 
«las dos», hasta (nes xeneraciones del Franquismu) un $60 \%$ pa castellanu, un I5 \% p'asturianu y un I8 \% pa «las dos».

La diferencia ente los datos de les dos encuestes nun s'esplica tanto por un desgaste d'una intensidá tala malapenes nuna década como pola supresión d'una opción d'abelugu como «mezcla» na que probablemente diben atopase bien una parte del $60 \%$ de supuestos castellano-falantes. Nun se trata con esto de faer de menos la validez de la encuesta (quiciabes más realista que la de 199I), sinón de rescamplar cómo una más esixente definición de lo que tien que s'entender como llingua asturiana (ensin l'abelugu d'esa «mezcla» que tamién ye asturiana nun sen idiomáticu ya identitariu) provoca una verdadera quiebra na concencia llingüística del informante.

Esto apurre un elementu de reflexón adicional sobro l'aparente caída na concencia llingüística ente 1991 y 2002: anque ente estes feches nun hai una alteración significativa na fala de la población, ye'l momentu del impactu de les primeres polítiques llingüístiques del gobiernu autonómi$\mathrm{cu}$, de puxu del movimientu reivindicativu y de consolidación nel imaxinariu social del modelu normativu o testual emerxente nel Surdimientu. Esto ye: d'una definición más terria de lo que ye d'entendese como asturianu na que les amestadures pierden llexitimidá. Con ello, los encuestaos evalúen les sos competencies llingüístiques al son d'esi modelu referencial emerxente col que, amás, van perder capacidá d'identificación una parte significativa d'ellos. Como corolariu positivu, puede deducise que les polítiques llingüístiques (meyores o peores) y el movimientu social en favor de la llingua acrediten un impactu real y significativu na concencia llingüística ciudadana.

Valgan de prueba los datos del estremu occidental d'Asturies. La llingua local, nun contestu típicu de transición, foi históricamente caracterizada como una variedá de gallegu, col que tipolóxicamente muestra mayores afinidaes y col que viniera siendo asociáu ensin mayor proble- 
matismu. L'apaición d'una reivindicación local d'inspiración galleguista nos ochenta afaló, por reacción, un esfuerzu discursivu del asturianismu oficial (y otros sectores menos sensibles coles llingües minorizaes) pa demostrar que la fala de la zona nun ye en realidá gallegu, sinón una llingua románica estremada y, poques gracies, asturiana dafechu. D’ehí resultaron unes fuertes polémiques mediátiques dende finales de los ochenta, correlatives nes encuestes con un progresivu esmucimientu dende una percepción más galleguizante en I99I escontra otra más asturianista en 2002. En I99I (con un 66,8 \% qu'asume falar un amiestu de gallegu y asturianu) un II \% consideraba falar una variedá de gallegu y un 9,5\% d'asturianu. En 2002, la primer opción baxa al 7,7 \% y la segunda xube hasta'l I6,3\%. La llingua sigue siendo la mesma.

Si'l debate mediáticu interfier l'análisis que fai'l falante de la so propia fala, ye importante dilucidar cómo se vivía la llingua en periodos previos a esti tipu d'alderiques actuales, cómo yeren les coses a lo primero. Y yelo porque esi imaxinariu xenuín entá recibíu bonalmente por bona parte de los falantes actuales sigue conformando'l fondu irreductible de la so concencia llingüística, desque estos ciudadanos, fora del actu puntual de cubrir una encuesta, rara vez problematicen en términos ideolóxicos los sos vezos cotidianos o s'enguedeyen en sutileces filolóxiques.

Si’l primer pasu nun análisis sociollingüísticu ye determinar cuántos falen asturiano y falar asturiano ye socialmente pertinente na midida que reflexe dalgún tipu de concencia diferencial que lo faiga relevante n'actos cotidianos ¿d'aú vien esta o cómo se articula primero de la difusión de discursos reivindicativos o ente los sectores más ayenos a los discutinios de los medios? Nuna situación de contactu diglósicu y amestadura, ¿cuála ye la riegla de midir que tien el falante común a la de decidir si ta más cerca del focu asturianu del espectru o de cullá de la llinde qu'intuitivamente lu estrema del dominiu del castellanu? 
Falamos de cómo percibía la llingua’l güelu del falante actual y cómo lo tresmitía al padre (la llingua y la so percepción) nun entornu familiar o comunitariu al abelugu de la presión oficial o de la retórica militante. Consideramos amás qu'esta percepción (validada nel día a día na so coesistencia contrastiva col castellanu) respuende a una esperiencia llingüística mui aquilatada nel tiempu: la caracterización qu’ofrez Martínez del paisaxe llingüísticu d'Uviéu na metá del xx nun difier de manera de la qu'ofrez Xovellanos dos sieglos primero, daqué de so significativo del grau d'equilibriu nel que discurrió a lo llargo de tou esti tiempu la relación contactual asturianu-castellanu, en términos de diglosia sostenible. Y ello, amás de recordar el ritmu lentu del desplazamientu llingüísticu n'Asturies hasta feches bien recién, suxur delles claves sociolóxiques que nun foron encetaes con decisión nin nos estudios sociollingüísticos nin nos discursos públicos sobro l'asturianu.

El reveláu de la percepción última del asturianu en dómines pasaes ye sicasí difícil por cuantes va siguir dependiendo d'imponderables. Nin siquier los relativamente abondosos testimonios metallingüísticos d'época son especialmente rescamplantes, poles sos ambigüedaes y zunes ideolóxiques. Pero, cuandoquier, puede albidrase qu'ha tener daqué tipu d'anclaxe na concencia histórica recibida al traviés de la esperiencia social de cada individuu. Les encuestes ofrecen nesti sen una imaxe creyible sobro la evolución del últimu mediu sieglu y de les claves del desplazamientu d'asturianu por castellanu nos años centrales del sieglu xx. El mio envís agora ye prospectar no que pudiera pasar en xeneraciones anteriores: na de los padres de los primeros asturianos que dexaron de falar asturiano o de tresmitilo espontáneamente a los sos fíos.

Voi basame nuna reellaboración de les encuestes de I99I y 2002 entecruzando los sos datos con otres variables sociales y demográfiques. Alvierto agora yá que va tratase d'un exerciciu inductivu de trazu gruesu que va entamase con un intentu d'homoxeneización de los criterios 
dispares remanaos nos dos trabayos, con una reducción por aciu de redondeos y medies aritmétiques sobro unos datos que, otra manera, danse na fonte orixinal con precisión desigual. El voluntarismu d'esta prospección presupón, como sospecharál llector, contrapuntiar la deficiente aproximación matemática a la prehistoria de la sociollingüística asturiana con delles intuiciones basaes en testimonios como los ufiertaos o n'apreciaciones suxetives fraguaes na vivencia llingüística particular de mio. Sía como quier, nun va interesar tanto la esactitú nos númberos como la identificación de situaciones y la detección de tendencies.

Como procedimientu xeneral, voi fundir los datos de les dos encuestes atendiendo a les feches de nacencia de los informantes y considerando criterios xeneracionales históricamente pertinentes. Va considerase una xeneración de Preguerra (nacíos dende antes del períodu I93I-I942/45, según la fecha ante quem de cada encuesta), la de Posguerra (ente 1942/45 y 1955/57); la del Desarrollismu (nacíos primero de I975/77) y la de la Transición y Autonomía (con posterioridá a 1977). Coles mesmes, voi centrame namá na variable "primera lengua», recurriendo a la media de les preguntes "primera lengua en la infancia» y «lengua familiar» de la encuesta de 2002 cola correspondiente de I99I; $y$, otramiente, fundiendo nuna media aritmética les respuestas «una mezcla» $\mathrm{y}$ «las dos» d'esta última, presuponiendo nos dos grupos una xenérica competencia billingüe. Los datos brutos per xeneraciones son los siguientes:

\begin{tabular}{l|c|c|c} 
& Castellanu & Asturianu & Les dos \\
\hline Preguerra & 39,5 & 36 & 24,5 \\
\hline Posguerra & 42,5 & 32,5 & 25 \\
\hline Desarrollismu & 46 & 21 & 30,6 \\
\hline Transición & 70 & 9 & 21
\end{tabular}


Resalvando les cifres concretes, ha notase que'l verdaderu esbarrumbe del asturianu produzse ente los nacíos en democracia, ente los fíos de los nacíos contra 1950, que ye la xeneración na que fraya la tresmisión interxeneracional. Ye en cierto la qu'acusa la paradoxa de francer el filu de la tresmisión llingüística y, per otru sitiu, activar el movimientu de reivindicación de magar los 70, de la qu'algama la so madurez y protagonismu públicu. Verifícase entós el principiu reactivu formuláu por Weinreich pa les llingües en contactu y la paradoxa que tanto presta sorrayar a los detractores de la normalización: que los que reivindiquen l'asturianu bien veces nun lo falen.

Hai, sicasí, matices interesantes nel análisis d'esta xeneración intermedia (la de la emigración a les ciudaes, trabayu nuna economía industrial y de servicios, accesu a mass media o mayor nivel educativu). $\mathrm{Na}$ tabla, lo llamativo nun ye tanto la medría estadística del castellanu monollingüe (a un ritmu constante respecto a xeneraciones anteriores) sinón la caída sópeta del asturianu nos años del Desarrollismu, que desplaza parte importante de los sos adscritos al grupu billingüe (que pasa d'un cuartu a un terciu de la población en pocos años). Esto nun suxur tanto un tayu radical na tresmisión familiar del asturianu (siquier, non más marcáu que nes xeneraciones previes) como un aumentu funcional del castellanu. Ello ye que los competentes n'asturianu van siguir siendo una mayoría con respecto a los monollingües en castellanu. Sumando les opciones «asturianu» $\mathrm{y}$ «les dos», les cifres resultantes seríen:

\begin{tabular}{l|c|c} 
& Solo castellanu & $\begin{array}{c}\text { Asturianu } \\
\text { (billingüe + monollingüe) }\end{array}$ \\
\hline Preguerra & 39,5 & 60,5 \\
\hline Posguerra & 42,5 & 57,5 \\
\hline Desarrollismu & 46 & $5 \mathrm{I}, 6$ \\
\hline Transición & 70 & 30
\end{tabular}


Esto ye: l'asturianu siguiría teniendo usu mayoritariu (siempre según niveles y rexistros) hasta la Transición, anque nel Desarrollismu carez d'una erosión del $6 \%$, que dobla'l $3 \%$ del cambiu xeneracional anterior. Pero a faer casu a pies xuntos de les dos tables, el castellanu monollingüe namá miedra nos niveles previos (un 3,5\%), siendo lo notable'l corrimientu de monollingües n'asturianu al grupu de los billingües.

Hai dellos otros aspectos suxestivos. Ente los nacíos esos años, hasta un Io \% defínense como falantes de castellanu y otra llingua, el mayor nivel d'ente tolos grupos d'edá. Trátase d'inmigrantes que lleguen a Asturies nel momentu de la industrialización, ente los que presumiblemente van tener más relevancia estadística gallegos y portugueses. L'amestar esti grupu al de monollingües castellanos (como fiximos) dexa faese una idea del impactu de los inmigrantes nel paisaxe llingüísticu asturianu metál sieglu xx, cuantimás considerando que tamién llegaron otros monollingües castellanos que sumen ensin más en mesmu grupu de castellano-falantes locales ${ }^{4}$.

Sía como quier, l'impactu d'esti colectivu nun paez repercutir de manera nin inmediata nin directamente proporcional nel retrocesu cuantitativu del asturianu, una y bones, considerando en xunto'l monollingüismu y el billingüismu con asturianu, el retrocesu global malapenes ye dalgo mayor que na xeneración precedente. Dicho otramiente: nes décades centrales del xx l'asturianu entá tien potencia social abondo pa percibir falantes que tendríen el castellanu o otres llingües nel so rexistru familiar. Daqué qu’intuitivamente sabíemos los nacíos nesti períodu podía tener aquí ciertu reflexu estadísticu: que fíos de castellanos, anda-

${ }_{4}^{4}$ En 2002, los inmigrantes faíen el I6,3\% de la población asturiana, el I3,4 \% procedente d'otres partes d'España y un 2,9 \% d'otros países. Pa la so encuesta, l'equipu de Llera Ramo tomó en cuenta un $70 \%$ de persones colos dos padres asturianos, I4,8 \% de families amestaes y $15 \%$ de padres inmigrantes (ibidem, páx. 28). Según esto, hasta un mínimu del $30 \%$ d'encuestaos dispunxeron d'una llingua non asturiana nel usu familiar. 
luces o gallegos tamién falaben un asturianu más o menos amestao con naturalidá y plena concencia, ensin que lo afalare presión institucional nenguna nin una actitú reivindicativa espresa. Por eso, ensin faer de menos los efectos al mediu plazu de la inmigración, la quiebra llingüística que va cadecer la xeneración posterior tien que s’esplicar más bien por otros factores, como la escuela o los medios.

¿D’aú sal esta potencia social del asturianu entá a mediaos del sieglu xx? ¿Cómo ye qu'acueye nuevos falantes ya inclusive ye quien a garrar concencia d'él mesmu y entamar un procesu d'autoafirmación a lo postrero del Franquismu si ye que daveres nun cuenta nin con sofitu institucional nin cola referencia de nengún discursu social esplícitamente favorable? La respuesta ye de so obvia: porque, a última hora, l'asturianu fálase, lo que quier dicir, en términos sociolóxicos, que se fala conscientemente, como una estratexa eficiente de socialización; lo que ye dicir en términos sociollingüísticos que, anque la so forma creciente d'esistencia social sía l'amestadura col castellanu, el falante tien una clara noción de diferencialidá, de que ye daqué distinto, d'aniciu distintu, col so propiu ámbitu y la so propia funcionalidá. Y esto ye asina porque, pola so propia esperiencia social, el falante nacíu naquellos años tien contactu con formes más puxantes d'asturianu y más impermeables a la castellanización que'l so propiu rexistru xeneracional, como les reconocibles nes xeneraciones precedentes.

L'asuntu ye entós cómo falaba la xente nacío primero de la xeneralización de la economía industrial y urbana, primero de la Guerra Civil, de les campañes d'alfabetización o del gran desenvolvimientu de los medios de comunicación. Estes xeneraciones tienen ruin reflexu nes encuestes de I991 y 2002, cuantimás considerando que pa les feches d'estos estudios yá tendríen atropao una esperiencia sociollingüística enforma más complexa y actualizada que tuvo qu'alteriar la percepción de la so llingua infantil, fayéndola de menos o idealizándola. Resalvando 
estos imponderables, vamos mirar de prospectar de cullá d'esi términu ante quem qu'afita en I93I la primera d'eses encuestes pentemedies del análisis de los sos númberos.

De tables anteriores entesácase una pauta de desplazamientu llingüísticu a un ritmu del $3 \%$ : ente les xeneraciones de Guerra y Posguerra (I93I-I945), el valumbu de monollingües en castellanu miedra nesta proporción (del 39,5\% al 42,5\%) y los d'asturianu mengüen de manera asemeyada (3,5\%, del $36 \%$ al 32,5\%), con una esencial coincidencia nos datos de los billingües $(24,5 \%$ y $25 \%)$. Polo tanto, como puede vese, a tomar como referencia'l conxuntu de falantes d'asturianu (monollingües o billingües) la diferencia ye tamién del $3 \%$ (del 60,5\% al $57 \%$ ). Sicasí, esto fai ver un procesu de desasturianización más lentu que’l propiu de castellanización, desque presupón una fase intermedia de billingüismu pela que pasa la población asturfalante primero de la so castellanización definitiva.

De primeres, podía pensase que nes décades inmediatamente anteriores el ritmu de desplazamientu llingüísticu nun fora esencialmente distintu. Per un sitiu, ye verdá qu'estos datos procedentes d'encuestes feches mui a posteriori favorecen estadísticamente al castellanu, desque tenemos que suponer qu'ente los encuestaos nacíos nos años de Preguerra dellos son tamién inmigrantes llegaos con posterioridá y non nativos d'Asturies. Esto ye: xente nacío al rodiu de 1920-30 que seique llega a Asturies contra 1950, pero que nun habíen computar nes estadístiques sociollingüístiques de la Preguerra asturiana, por más que consten como población asturiano nes encuestes futures de I991 y 2002. Coles mesmes, queda ensin contabilizar población nativo y asturfalantes que pelos mesmos años y anteriores emigren masivamente a América (nel primer terciu del sieglu) o a Europa (na Posguerra) y que lóxicamente nun remanecen nes encuestes feches n'Asturies décades depués. Sicasí, el primer terciu del sieglu xx, de relativa prosperidá n'Asturies, ha vese tamién como un 
momentu de puxu na institucionalización del castellanu pente medies d'un sistema educativu espansivu, de los medios de comunicación, de l'articulación de movimientos sociales y políticos, normalmente con proselitismu en castellanu, ateneos culturales y obreros, etc. Ha suponese, polo tanto, que ciertu porcentaxe de población pasa a acoyese al castellanu como principal llingua referencial.

Con estes, vamos da-y verosimilitú pal primer terciu del xx a un escenariu con un ritmu de castellanización del $3 \%$, entendiendo qu'esi ye l'aumentu per cada quince años del grupu de castellanofalantes monollingües. Sicasí, vamos considerar al par una desasturianización daqué más lenta en comparanza cola que se detecta en pasando la Guerra, sobro la base (yá contrastada enantes) de qu'esi $3 \%$ provién non directamente del grupu d'asturianu monollingüe, sinón del total universu asturfalante. Desque asina, vamos calibrar pa esi mesmu trechu temporal un I, $5 \%$ de reducción de la base social tanto de monollingües como de billingües con asturianu. Yá viemos de riba cómo, en realidá, nin siquier la entrada de población nuevo implicaba un descensu estadísticu del asturianu na mesma proporción, esto ye: que l'aumentu evidente del castellanu nun trai de so una mengua equivalente nel asturianu.

En resalvaes estes convenciones más o menos argumentables y aplicando esa movición del $3 \%$ cada quince años, puede postulase pante los nacíos contra 1900 esti panorama:

\begin{tabular}{c|c|c} 
Castellanu & Asturianu & Les dos \\
\hline 33,5 & 39 & 27,5
\end{tabular}

Con un 66,5\% duechu n'asturianu y un 6r \% duechu en castellanu. L'asturianu atecha entós hasta un mínimu de dos tercios de los padres y güelos de los nacíos nes décades posteriores a la Guerra Civil, representando una clave importante de la so esperiencia familiar y na formación 
de la so concencia llingüística, entá nun momentu de creciente escayencia de la llingua.

En comparanza, al son de los datos directos de la encuesta xenuina de Llera de 199i (la de más fondura histórica), la evolución ente los dos grupos d'edá más antiguos sería esta:

\begin{tabular}{l|c|c} 
& $\begin{array}{c}\text { Castellanu } \\
\text { monollingüe }\end{array}$ & $\begin{array}{c}\text { Asturianu }+ \\
\text { billingüe }\end{array}$ \\
\hline Nacíos primero de I93I & 25 & $75(5 \mathrm{I}+24)$ \\
\hline Nacíos primero de I945 & 33 & $67(39+28)$
\end{tabular}

En xeneral, los datos directos de Llera apurríen en I99I cifres enforma más positives pal asturianu y, pela contra, un ritmu de desplazamientu llingüísticu superior al barruntáu aquí. Ello ye que Llera suxur pante I93I-I945 una situación casi hermana de la que postulemos pa I900. Esto ye: cocinando los sos propios datos, contemplamos la posibilidá d'alantrar nuna xeneración l'avance de la castellanización, albidrándoy sicasí un ritmu menor, del $3 \%$ cada quince años y non del $8 \%$ que detecta la encuesta de Llera pal mesmu forcu temporal. Faise bien razonable (polo dicho enantes) una revisión al alza de los datos sobro'l númberu real de asturfalantes na Preguerra, pero abúltanos un tanto escesivo pa la socioloxía asturiana del primer terciu del sieglu xx un retrocesu llingüísticu de casi una décima parte de la población cada quince añoss.

s Puede en tou casu considerase que los nuesos cálculos refiérense en realidá a posibles magnitúes mínimes de presencia social del asturianu. La esplicación a estos supuestos desaxustes podía ser l'apuntada enantes: computen como castellano-falantes persones nacíes fora d'Asturies nestos años pero solo asitiaes nel país na época del Desarrollismu posterior. Otro ye que falar de mayor o menor ritmu de castellanización en cuantes a porcentaxe de falantes implicaos nun diz nada de la intensidá d'esa castellanización, esto ye: del grau d'acastellanamientu de la fala real, variable que nun ye a captase con una pura operación matemática. Arriendes d'ello, tamién entra en xuegu un 
A la de matizar les estimaciones espuestes sobro datos d'encuestación directa, pueden amestáse-y al análisis otres evidencies como les qu'ufierten los censos del momentu ${ }^{6}$ y que, nel so casu, podíen valir p'afinar de manera indirecta les ponderaciones cuantitatives anteriores. Por casu, el censu oficial d'Asturies de 1900 recueye, pa un total de 627.069 habitantes, un 23,I6 \% de población urbano frente a un 76,84 \% rural, valores prácticamente inversos a los actuales. Esta distribución asemeya daqué a los porcentaxes de monollingüismu castellanu y de falantes d'asturianu, pudiendo albidrase tamién que la prefigura, anque con importantes matices. De primeres, los conceyos urbanos (Uviéu, Xixón, etc.) nun son namá urbanos, desque inclúin un porcentaxe importante de población y territoriu rurales, mesmo que los teníos de rurales pueden contar con villes de cierta estructura urbana, onde podíen entrar fácil nes sos pequeñes élites los vezos castellanizantes, como apunten dende’l xIX autores como Caveda o Munthe. Tampoco nun ye de presuponese que la población urbano de la dómina tuviere castellanizao dafechu, una y bones casi un sieglu depués entá se constata un panorama enforma más matizáu, como viemos. El tipu de sociedá reflexada en censu de 1900 lo que sí presupón ye un nivel altu d'interacción y dependencia de los centros urbanos (focos virtuales de castellanización) y la so redolada rural (depositaria de la tradición llingüística asturiana) que nos afala a supo-

argumentu psicolóxicu a la de ponderar estos datos: nel marcu apuntáu de castellanización, la creciente institucionalización del castellanu nun trai darréu por fuerza la castellanización inmediata de la fala en tol so espectru de rexistros, pero seguramente sí una clara percepción psicolóxica de la so espansión y, a última hora, del so trunfu social, y eso ye lo que podía tar reflexando la opinión de los informantes de Llera nacíos y socializaos primero de la Guerra Civil, falaren como falaren. Por poner un casu: la constitución republicana de 193I ye la de proclamar, pela primer vez na historia, el carácter oficial del castellanu, nun marcu ideolóxicu novedosu, aperturista y democráticu, qu'espeya, otra manera, el discursu intelectual sobre la llingua ellaboráu con precuru dende'l noventayochismu cola so espresión más acabada na obra de Menéndez Pidal. Naturalmente, esi trunfu social del castellanu fíxose más evidente, pa les mesmes persones, a lo llargo de les décades vinientes hasta la fecha de les encuestes.

${ }^{6}$ http://www.ine.es/inebaseweb/pdfDispacher.do?td=69583\&ext=.pdf 
ner una fuerte presencia del asturianu na ciudá, anque tamién una fácil infiltración de les tendencies castellanizantes burgueses na so contorna rural más inmediata. Sía como quier, siguiendo cola hipótesis reactiva pa en situaciones de contactu, apunta un fuerte choque de llingües nos núcleos urbanos y unes condiciones afayadices pal caltenimientu d'una concencia diferencial.

Un aval asemeyáu puede afayase na estructura económica del país. $\mathrm{Na}$ economía asturiana del momentu predomina l'agricultura y la ganadería (un 82,I \%) frente a actividaes urbanes como industria $(8,2 \%)$ o servicios (9,7\%). Estos datos animen otra vuelta a amontar el datu de competencia n'asturianu, tradicionalmente la llingua casi privativa nesti sector (que serál gallego-asturianu na so zona). Pela contra, asumiendo'l castellanu como llingua dominante en terciariu, ye d'atribui-y un colchón estadísticu averáu al ıo \% de la población. Cuandoquier, esti ıo \% ye'l nucleu duru de la castellanización, quiciabes el so focu principal en términos sociolóxicos. Otra manera, ha pensase qu'esti io \% del total de la población asturiana concéntrase xeográficamente precisamente en centros urbanos, de cuenta que nes zones inmediatamente contigües a estos (que son, por definición, les más poblaes) el so impactu cuantitativu y estadísticu miedra sensiblemente y con él la capacidá d'influencia llingüística que se-y presupón pente otros grupos poblacionales. A otru sen, el mundu mineru (que concentra esi $8,2 \%$ de población industrial) vieno siendo daveres un potente nucleu de falantes d'asturianu, na so mayor parte procedentes del mundu rural y bien xuncíos a él. La cultura minera ye seique ún de los principales motores d'absorción llingüística de la población inmigrao a les cuenques, onde l'asturianu fai de facto de llingua de clase nuna contorna sociolóxica fuertemente ideoloxizada. Coles mesmes, pa Diego Catalán la esistencia de centros industriales na redolada d'Avilés tamién yera un factor de propagación escontra l'occidente de soluciones estándar centrales, como modelu localmente más prestixosu que'l propiu castellanu. 
En cuantes a alfabetización, el censu de 1900 namá estrema los que $s a-$ ben lleer y los que saben lleer y escribir, y amuesa una tasa d'alfabetización del $46,7 \%$ contra un $55,3 \%$ d'analfabetos, datu importante desque la castellanización nestos años fíase mayormente a la escolarización. Que nun contestu talu haya hasta un $40 \%$ de monollingües y hasta un 66,5 de competentes n'asturianu faise una estimación de mínimos bien de prestar, sobro manera cruciando'l datu col relativu a la población rural, la que mayormente cadez del analfabetismu.

Desque asina, vamos volver a una observación anterior. En 1946 Catalán y Galmés daben cuenta de la penetración del estándar asturianocentral na fala oriental, inclusive con más fuerza referencial que'l castellanu, como vien de vese tamién al occidente. Estos autores focalicen el fenómenu entre los más mozos de llugares bien comunicaos con centros urbanos y vencéyenlu a un ciertu nivel formativu, que, en xeneral, ye de suponese non mui p'acullá de los estudios primarios. Un mozu de 20 años sería nacíu contra I925, fíu de daquién nacíu contra I900, el nuesu añu de referencia. Desque asina, ¿cómo ha esplicase'l sentíu d'esta observación?

Dende lo postrero del XIx dase un xorrecimientu de la lliteratura n'asturianu, popularizada al traviés de periódicos, cartafueyos y llibrinos o en recitales y representaciones teatrales. Ye una lliteratura bien desigual en términos estéticos pero, sicasí, bien popular que, otra manera, establez un ciertu canon castizu, un prototipu identitariu de lo asturiano al son del costumismu del xIx y el rexonalismu de les primeres décades del xx. Les tiraes d'estos cartafueyos raramente baxaben de los 6.00o exemplares (García, 2004: 193), que pa una población con 46,7 \% d'alfabetización (menos de 300.000 persones) implica un nivel de difusión bramente notable y una motivación nada ruina a la de faelo n'asturianu.

Nun ye d'estrañar entós que los mozos de 1946, amás de ser falantes nativos de dalguna variedá local, contaren cola referencia d'un modelu 
llingüísticu más o menos estandarizáu y dotáu de ciertu prestíu; un refuerzu a la so concencia llingüística que-yos venía dau precisamente pol so nivel básicu d'instrucción y d'acordies con unes pautes socioculturales que yeren, daquella, netamente urbanes. Too ello ensin resalvar el pesu demográficu del asturianu, entá mayoritariu naquellos años. Asina, los nacíos entós, anque socializaos nun contestu (el de los años 50-60) yá bien distintu al tradicional, entá teníen bonos argumentos pa sofitar una sólida concencia llingüística. De magar entós, los carreros siguíos pola sociedá asturiana sol Franquismu nun favorecieron l'afitamientu y el desenvolvimientu de la llingua. Sicasí, ye precisamente la solidez d'esta concencia recibida la que dexa esplicar, por reacción énte la redoblada presión castellanizante, la eclosión del movimientu llingüísticu asturianu de los 70, cuando la xeneración mentada algama la madurez.

L'Asturies moderna vien filando les sos propies narratives sobro'l procesu de revitalización de la llingua como llexitimación de les distintes posiciones, pronormalizadores o contraries. Nesti esquema, el principal interés radica n'estimaciones puramente cuantitatives, sobro cuántos tán a ún o otru llau o cuántos falen o non asturianu. La sociollingüística vien averándose a estes cuestiones con más o menos alvertimientu y tien aportao una panorámica fiable del paisaxe llingüísticu actual. La falta ye'l nun tenese propuesto dir más acullá d'un marcu discursivu concebíu pal debate públicu y non tanto pa la plena comprensión científica de les claves de la nuesa realidá social y llingüística. Sicasí, un averamientu puramente superficial fai alvertir na vivencia llingüística de los asturianos delles dinámiques espontánees de gran caláu sociolóxicu que cuestionen dellos tópicos del debate. Nin l'asturianu llegó hasta güei como daqué quito de toa funcionalidá social, malapenes alimentao por complots políticos, mimetismos o postureos, nin la potencia actual de la so reivindicación respuende ensin más a la sufrida clarividencia de ciertes élites asturianistes. L'asturianu tuvo ende dende siempre y emite lluz propio, avenible a ser interpretada. Si'l desdeñu d'unos nun quixo ver esa lluz o 
la llistura d'otros quixo dotase d'un brillu inesperáu colos sos rellumos tien poco interés tanto pa la sociollingüística como pa definir una estratexa normalizadora bramente eficaz.

\section{BIBLIOGRAFÍA}

Catalán (i956) = Diego Catalán Menéndez-Pidal, "El asturiano occidental», Romance Philology, Io (1956), páxs. 7I-92.

Catalán \& Galmés (i946) = Diego Catalán Menéndez-Pidal \& Álvaro GalMÉs DE Fuentes, "Un límite lingüístico», Revista de Dialectología y Tradiciones Populares, 2 (1946), páxs. 196-239.

Caveda (I939) = José Caveda y Nava, Colección de poesías en dialecto asturiano, Oviedo, ı839. [Ed. Facs. Uviéu (Alvízoras), ı989].

ANDRÉs (I993) = RAMÓN D’ANDRÉs DÍAZ, Encuesta sociollingüistica nuna parroquia asturiana (Deva-Xixón), Uviéu (Universidá d'Uviéu), I993.

ANDrÉs (2002) = RAMÓN D’ANDrÉs DíAZ, «L'asturianu urbanu mínimu. Delles hipótesis», Lletres Asturianes, 8I (2002), páxs. 2I-38.

Fernández Lorences (2OII) = TAResa Fernández Lorences (2OII), "Alternancia y mestura de códigu asturianu / español: problemes nel determín de les fronteres llingüístiques», en Ramón d'Andrés (ed.), Lengua, ciencia y fronteras, Uviéu (Universidá d'Uviéu), 20II, páxs. I9I-2II.

García Fernández (2004) = Antón García Fernández, «Public lecteur, diffusion et thématique dans la litterature en Asturien de nos jours", Lengas, 56 (2004), páxs. 187-208.

García García (2008) = Serafina García García, «El asturiano en la obra de Ramón Menéndez Pidal (I869-I968)", en Xulio Viejo Fernández, Cien años de filoloxía asturiana (1906-2006), Uviéu (Alvízoras \& Trabe), 2008, páxs. 43-68.

González Riaño (1994) = Xosé Antón González Riaño, Interferencia lingüistica y escuela asturiana, Uviéu (Academia de la Llingua Asturiana), I994.

González Riaño \& García Arias (2006) = Xosé Antón González Riaño \& Xosé Lluis García Arias, Estudiu sociollingüisticu de Lleón, Uviéu (Academia de la Llingua Asturiana), 2006.

Jovellanos (198I) = Gaspar Melchor de Jovellanos, Cartas del viaje de Asturias, Salinas (Ayalga), I98I. 
Llera RAMO (1993) = FranCISCO LleRA RAmo, Los asturianos y la lengua asturiana. Estudio sociolingüistico para Asturias, I99I, Uviéu (Principáu d'Asturies), I993.

Llera Ramo \& San Martín (2002) = Francisco Llera Ramo \& Pablo San MarTín Antuña, II Estudio sociolingüistico para Asturias, Uviéu (Academia de la Llingua Asturiana), 2002.

Llera Ramo \& San Martín (2005) = Francisco Llera Ramo \& Pablo San Martín Antuña, L'asturianu en Xixón. Primer encuesta sociollingüística municipal, Xixón (Ayuntamientu de Xixón), 2005.

Martínez (1967) = Josefina Martínez Álvarez, Bable y castellano en el concejo de Oviedo, Oviedo (Universidad de Oviedo), 1967.

Menéndez (1963) = Manuel Menéndez García, El Cuarto de los Valles (un habla del occidente asturiano), Oviedo (Instituto de Estudios Asturianos), 1963.

Munthe (1987) = Åke Wilhelmson Munthe, Anotaciones sobre el habla popular de una zona del occidente de Asturias, Uviéu (Universidá d'Uviéu), I987.

Rodríguez-Castellano (1954) = Lorenzo Rodríguez-Castellano, Aspectos del bable occidental, Oviedo (Instituto de Estudios Asturianos), I954.

Rodríguez Valdés (2009) = Rafael Rodríguez Valdés, Discursos llingüísticos y movimientu obreru na Asturies decimonónica, Uviéu (Trabe), 2009.

SADEI (1984) = Asturias. Segunda encuesta regional, Oviedo (Principado de Asturias / Sociedad Asturiana de Estudios Económicos e Industriales), I984.

Viejo Fernández (2002) = Xulio Viejo Fernández, "Sociollingüística y lliteratura asturiana nel sieglu XVII», Actes del ciclu de conferencies "La Lliteratura Asturiana nel IV Centenariu d'Antón de Marirreguera», Uviéu (Principáu d'Asturies), 2002, páxs. 27-58.

Weinreich (I974) = Uriel Weinreich, Languages in contact. Findings and problems, Paris/The Hague (Mouton), I974. 
